\title{
IMPACTO EN LA SOCIEDAD A TRAVÉS DE LA RECUPERACIÓN DE LAS ÁREAS VERDES
}

AUTORES: Marleydis Claudia Solís Maldonado ${ }^{1}$

Kirenia Maldonado Zuñiga ${ }^{2}$

Dunia Lisbet Domínguez Gálvez ${ }^{3}$

Verónica Jazmín Pionce Andrade ${ }^{4}$

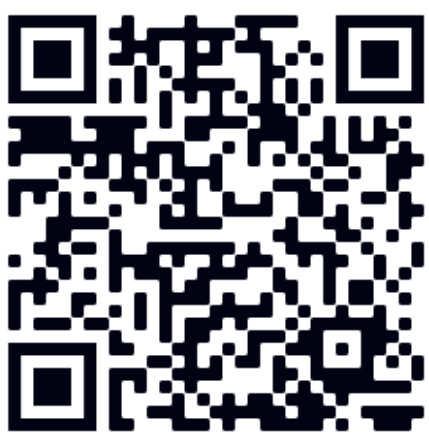

DIRECCIÓN PARA CORRESPONDENCIA:solis-marleydis5181@unesum.edu.ec

Fecha de recepción: 12/08/2019

Fecha de aceptación: 07/11/2019

RESUMEN

La importancia de esta investigación es dar a conocer la recuperación de las áreas verdes en las zonas urbanas y su impacto en la sociedad. Estas áreas en las ciudades reducen los niveles de contaminación. El objetivo de este trabajo es contribuir a la recuperación de áreas verdes urbanas, estas aportan beneficios como, combatir el estrés, la depresión, desarrollo del cerebro, reduce las enfermedades respiratorias, aumenta la actividad física y facilita la interacción social. Un grupo de estudiantes de la Universidad Estatal del Sur de Manabí con el apoyo de la ciudadela seis de agosto del cantón Jipijapa, lograron la renovación de las áreas verdes del parque de esta ciudadela, se desarrolló un trabajo creativo para la recuperación y el embellecimiento, se creó un ambiente agradable y natural. Los métodos utilizados son: del nivel teórico, histórico - lógico y análisis síntesis, del nivel empírico: observación, entrevista y revisión bibliográfica. Los resultados brindaron un conocimiento adecuado sobre la importancia del cuidado de las áreas verdes en los parques, se fomentaron hábitos de conciencia ambientalista en las personas que habitan en la comunidad para un buen vivir sano. Se concluye con la reforestación de las áreas verdes en su totalidad. El impacto en la población urbana fue significativo, la población urbana es amante de los espacios abiertos como parques y jardines. Las capacitaciones favorecieron en el cuidado y preservación. Las personas que están al cuidado de este sitio, enseñan a los niños y jóvenes a proteger las áreas verdes y al medio ambiente.

PALABRAS CLAVE: bienestar; medio ambiente; protección; salud; sostenibilidad.

\footnotetext{
${ }^{1}$ Estudiante de la Carrera Medio Ambiente. Universidad Estatal del Sur de Manabí, Manabí Ecuador. Solismarleydis5181@unesum.edu.ec

2 Máster en Ciencias de la Educación. Universidad de Ciencias Pedagógicas de Holguín, Holguín, Cuba. kirenia.maldonado@unesum.edu.ec

${ }^{3}$ Ingeniera en Procesos Agroindustriales, Universidad Oscar Lucero Moya de Holguín. Holguín - Cuba. dldominguez1006@gmail.com

${ }^{4}$ Magíster en Gestión Ambiental Universidad Técnica Estatal de Quevedo, Quevedo - Ecuador. verónica.pionce@unesum.edu.ec
} 
Marleydis Claudia Solís Maldonado, Kirenia Maldonado Zuñiga, Dunia Lisbet Domínguez Gálvez...

\section{IMPACT ON SOCIETY THROUGH THE RECOVERY OF GREEN AREAS}

\section{ABSTRACT}

The importance of this research is to publicize the recovery of green areas in urban areas and its impact on society. These areas in cities reduce pollution levels. The objective of this work is to contribute to the recovery of urban green areas, these provide benefits such as combat stress, depression, brain development, reduce respiratory diseases, increase physical activity and facilitate social interaction. A group of students from the South Manabí State University with the support of the Citadel on August 6 of the canton of Jipijapa, managed to renovate the green areas of the park of this Citadel, developed creative work for recovery and beautification, a pleasant and natural environment was created. The methods used are: from the theoretical, historical - logical and analysis - synthesis level, from the empirical level: observation, interview and bibliographic review. The results provided adequate knowledge about the importance of caring for green areas in parks, environmental awareness habits were fostered in people who live in the community for a good healthy life. It concludes with the reforestation of the green areas in their entirety. The impact on the urban population was significant, the urban population loves open spaces such as parks and gardens. The trainings favored in the care and preservation. The people who take care of this site teach children and young people to protect green areas and the environment.

KEYWORDS: environment; Health; protection; sustainability; wellness.

\section{INTRODUCCIÓN}

La Organización Mundial de la Salud recomienda de 10 a 15 metros cuadrados de superficies de áreas verdes por habitante, pero aún no se cumple ese objetivo en muchas partes del mundo por diversas razones. Las áreas verdes urbanas representan un enfoque planificado, integrado y sistemático del manejo de árboles, arbustos y otro tipo de vegetación en centros urbanos. Por lo tanto, el establecimiento de áreas verdes urbanas requiere de una amplia planeación con la meta de lograr beneficios ambientales y sociales para sus habitantes. En consecuencia, el establecimiento de las áreas verdes urbanas implica actividades y enfoques interdisciplinarios.

Los beneficios sociales de las áreas verdes urbanas están relacionados con la salud pública, la recreación, factores estéticos y bienestar general, pueden hacer del ambiente urbano un lugar más placentero para vivir, trabajar y utilizar el tiempo libre; los beneficios ambientales incluyen el control de la contaminación del aire y ruido, la modificación del microclima y un realce del paisaje con impactos positivos en la psique humana y la educación, hacen amplias contribuciones a la vitalidad económica de una ciudad, vecindario o unidad habitacional. También proporcionan un hábitat para la vida silvestre, control de la erosión.

En la Constitución Política de la República del Ecuador (2008), en el Título VII del Régimen del Buen Vivir, toma como parte de los derechos de los ciudadanos lo que es dotar de áreas verdes para mejorar la calidad de vida de las personas.

En Manabí y muy particularmente en el cantón Jipijapa, el espacio público es un espacio para todos, se presenta como un elemento ambiental activo en el ecosistema, realizando una serie de funciones que son verdaderos servicios para comunidad de Jipijapa.

El concepto de parque como espacio público se puede definir como el enlace de relaciones de la comunidad, donde se producen los encuentros entre sus habitantes, por ello es necesario embellecer 
sus espacios físicos para lo cual se hace preciso realizar actividades que conlleven a mejorar el ambiente en el parque de la ciudadela 6 de Agosto del cantón Jipijapa, para que los ciudadanos como padres de familia, niños(as) y jóvenes puedan recrearse y disfrutar sanamente dándole un mejor uso.

La situación en el parque de la ciudadela 6 de Agosto de Jipijapa, era un poco deprimente porque estaba con hierbas, basuras, desechos, carecía de pintura y totalmente abandonado, lo que hace que la comunidad en general no se sienta a gusto. Es evidente el estado de deterioro y abandono en el que se encuentran sus instalaciones, situación que da lugar, a la generación de una aguda problemática ambiental y sanitaria dentro de las mismas y por consiguiente, el detrimento de la calidad del parque.

Los estudiantes de nivelación de la UNESUM del curso 9 A, contribuyeron en la recuperación del parque en todas las áreas, limpieza, organización, pintando, sembrado y cuidado de áreas verdes, dejando un entorno agradable a la vista de la comunidad y aumentando cada día las visitas al parque de las personas.

\section{DESARROLLO}

Las áreas verdes en las zonas rurales se convierten en auténticos pulmones que ayudan a reducir la contaminación del aire. Los parques, jardines y arboledas forman pequeños oasis en medio del hormigón y el asfalto que además regulan la temperatura y la humedad del ambiente. Estas producen oxígeno, filtran la radiación e incluso reducen los ruidos ocasionados por el tráfico. También ofrecen un ecosistema urbano que ayuda a la conservación de la biodiversidad.

Otra razón para afirmar la importancia de las áreas verdes es el bienestar que aportan, está demostrado que reducen el estrés y ayudan a las personas a conectarse con la naturaleza. La contemplación del paisaje y de un entorno natural relaja, fomenta la creatividad y las capacidades afectivas, y reduce la angustia. Además de ayudar a tener una buena salud emocional, los entornos naturales también permiten que sus habitantes se sientan mejor físicamente. Vivir rodeado de espacios verdes y parques ánima a la población a salir a la calle, a pasear e incluso a practicar deporte.

Varios estudios demuestran que los espacios verdes ayudan a las relaciones sociales, a que las personas se reúnan en este tipo de espacios, realicen actividades al aire libre y formen un sentimiento de comunidad.

Se crea una conciencia ambiental en los ciudadanos de la mejor manera de sensibilizarse con el medio ambiente es estar en contacto con la naturaleza. En las ciudades, donde impera el asfalto y el hormigón, el contacto cotidiano y la interacción con los espacios verdes del entorno que los rodea, los hace más conscientes de la importancia de cuidar las áreas verdes. Para los niños, vivir cerca de parques y arboledas es mejor que una clase de educación ambiental. Lo integran como parte de su vida.

Desde un enfoque positivo, los entornos naturales fomentan la creatividad, las capacidades mentales y afectivas, por lo que las zonas verdes urbanas ayudarían a mejorar la longevidad y la calidad de vida de sus habitantes. Puesto que se ha demostrado que el contacto con la naturaleza beneficia a largo plazo, los parques, jardines y pequeñas arboledas son auténticos y valiosos recursos para mejorar la salud y el bienestar. 
Marleydis Claudia Solís Maldonado, Kirenia Maldonado Zuñiga, Dunia Lisbet Domínguez Gálvez...

Todos los estudios indican que una de las cosas más importantes a la hora de crear conciencia ecológica es poder vivir la naturaleza. Da igual que sea en casa, en parques o en la montaña, vivir la naturaleza y estar en contacto con ella es un factor fundamental para que las personas tomen conciencia para restaurar y conservar las áreas verdes. Explicándolo su importancia para que los niños entiendan el porqué de conservar la naturaleza, vivirla y amarla, por ejemplo con las zonas verdes urbanas, les ayuda a crear esa conciencia.

Es de suma importancia que las autoridades y la comunidad en general cuiden y le den mantenimiento al parque para que no pierda la naturaleza y la esencia de su belleza.

Las Áreas Verdes Urbanas están creadas y construidas para el sano esparcimiento y bienestar de las personas, es por ello que mediante la investigación realizada en este trabajo, se pretende lograr un manejo integral de las áreas verdes del parque de la ciudadela 6 de Agosto. Para su mantenimiento y acondicionamiento. Es por eso que la investigación está elaborada con la finalidad del proceso de mejoramiento de las áreas verdes para que aporten con el buen vivir de los ciudadanos.

El objetivo principal fue recuperar las áreas verdes y embellecer el parque de la ciudadela 6 de agosto del cantón Jipijapa, limpiar la maleza y la basura que se encuentra en el parque de la ciudadela 6 de Agosto del cantón Jipijapa, contribuir con la siembra de árboles y flores (reforestar el área), pintar el parque con colores vivos para que se vea llamativo y a su vez que las personas puedan disfrutar en un ambiente agradable.

Este trabajo se ha realizarlo con el fin de hacer una buena obra en el cantón de Jipijapa, colaborar con un espacio agradable para las personas que quieran pasar un rato en el parque del barrio 6 de Agosto, debido a esto surgió la idea de sembrar flores y árboles, a su vez limpiar los residuos que contaminan el medio ambiente.

Los arboles desempeñan un papel muy importante en el planeta, estos cumplen funciones muy importantes para la vida de todos, ayuda purificando el ambiente y transformando el dióxido de carbono en oxígeno. Por ello es necesario apoyar la defensa del medio ambiente con la limpieza de desechos tóxicos como basura orgánica e inorgánica del parque de esta manera tener un ambiente saludable.

Se da a conocer la importancia del cuidado al medio ambiente dando charlas a las personas de esta comunidad para que tomen conciencia y ayuden con el cuidado del mismo de esta manera se contribuye con el cuidado del medio ambiente y de la salud de las personas.

Cuidar y mantener las áreas verdes mejora el desempeño de la cuenca hidrográfica, protegiendo al mismo tiempo el suelo de la erosión. Es importante tomar medidas para la reforestación de áreas verdes y crear áreas recreativas.

Las plantaciones de árboles producen resultados positivos, por los bienes que se producen y por los servicios ambientales que prestan. La reforestación es una actividad benéfica sus componentes contemplan la siembra de árboles para producción o para proteger el medio ambiente tienen impactos ambientales positivos siempre que se realice con especialistas en el tema. Las plantaciones y la reforestación de las tierras deterioradas y los proyectos sociales de siembra de árboles producen resultados positivos por los bienes que se producen y por los servicios ambientales que prestan.

12 UNESUM-Ciencias. Publicación cuatrimestral. Vol. 3, Año 2019, No. 3 (Septiembre - Diciembre) 
Para fomentar la sensibilización ambiental es necesario impulsar la acción ciudadana en defensa del medio ambiente, participando en acciones de recuperación y mantenimiento, incentivando la participación social y promueve la educación ambiental. Las reforestaciones participativas son plantaciones organizadas por asociaciones de voluntariado ambiental, centros educativos, ayuntamientos, etc. con el objetivo de mejorar, restaurar y conservar áreas verdes.

¿Qué es el medio ambiente?

Es el área condicionada para la vida de diferentes seres vivos donde se incluyen elementos naturales, sociales, así como también componentes naturales; como es el suelo, el agua y el aire.

Como parte de los seres vivos, se incluyen todas aquellas especies que llevan consigo acciones que demuestran vida; así como también las plantas; animales y seres humanos que llevan a su vez, los elementos simbólicos que hacen parte de su estructura completa y que se integran dentro del medio ambiente.

El medio ambiente es el entorno centrado en la biodiversidad de especies, donde se incluyen elementos naturales y artificiales que se relacionan entre sí; y que pueden verse modificados a partir del comportamiento humano.

¿Qué elementos conforman el medio ambiente?

Para conocer todos los conceptos que incluye el medio ambiente, es necesario abarcar lo que es el ecosistema, pues se traduce en el conjunto que une los factores bióticos de una región con los factores abióticos del medio ambiente; haciendo que se cree una comunidad de seres vivos con todos los elementos vitales relacionados entre sí.

Es necesario detallar el significado de la ecología, que no es más que otra noción bien determinada del medio ambiente; pues es la disciplina encargada de estudiar la compenetración entre los seres vivos y todo lo que le rodea.

La importancia del medio ambiente viene está dada a que es la fuente completa de la vida en todas las generaciones de especies existentes. Dentro de este entorno global se obtienen elementos para el sustento de la vida.

El medio ambiente es el hogar de todos, pero también depende de las acciones del ser humano. Al hacer mal uso de cada uno de los recursos naturales que en él se encuentran; las consecuencias de los daños se verán en tiempos futuros, poniéndolo en peligro y con muchas carencias que se evitarían si las acciones fueran en favor del ecosistema.

Al tratarse del entorno en el que se vive; se deben brindar efectos que colaboren con la recuperación y cuidado de las áreas verdes en las zonas urbanas, se considera importante dado a que es el entorno en que se vive, lo que requeriré mantenerlo en excelentes condiciones para seguir en el desenlace de todas las etapas de la vida con confort y con las áreas verdes que se necesitan en las zonas urbanas.

Es imprescindible el cuidado de las áreas verdes, así se contribuye a la protección del medio ambiente, evitar por completo todas esas acciones que tienen una repercusión que afecta con su vitalidad y conservación.

Los beneficios de limpieza en los parques favorecen su cuidado y permite el disfrute de la recreación sana. Estos brindan una salud física, mental y social para una mejor calidad de vida. Promover la conservación de las áreas verdes, poder relacionarnos aún más con la naturaleza, 
Marleydis Claudia Solís Maldonado, Kirenia Maldonado Zuñiga, Dunia Lisbet Domínguez Gálvez...

respirar aire puro entre otras actividades. Poder lograr espacios para el uso cotidiano de los ciudadanos es una labor de todos los días y tarea de todos.

Beneficios de las zonas verdes: combate el estrés y la depresión, si a menudo te sientes con mucho cansancio y sin ánimos, la medicina que te hace falta es recrearte en la naturaleza, desarrollo del cerebro, reduce las enfermedades respiratorias, aumenta la actividad física, facilita la interacción social.

Fue evidente el estado de deterioro y abandono en el que se encontraba el parque de la ciudadela 6 de Agosto del cantón Jipijapa, situación que da una aguda problemática ambiental y sanitaria dentro de las mismas, por consiguiente, el detrimento de la calidad del parque era fatal, por lo que surgió la idea de restaurarlo y capacitar a la comunidad cercana con la importancia de las áreas verdes dentro de las zonas urbanas, con este conocimiento pueden cuidar el parque, estas capacitaciones les permitieron asumir un verdadero papel de preservadores del medio ambiente, y más aun de las áreas verdes que los rodean.

Se logró recuperar las áreas verdes del parque de la ciudadela 6 de Agosto del cantón Jipijapa, se desarrolló el trabajo de forma creativo, se adquirieron las plantas para el embellecimiento, se limpió la maleza y se recogió la basura, se realizó la siembra de árboles y flores las que reforestaron el área, se pintó el parque con colores vivos y llamativos los cuales llamaron la atención de las personas de la comunidad, las que se vieron disfrutar en un ambiente agradable y natural.

Fue importante estimular la participación y el interés de la comunidad para incrementar recursos con destino a mejorar el parque, realizar la siembra de plantas con la colaboración de los vecinos, capacitar al personal encargado del mantenimiento de las áreas verdes urbanas, en lo que respecta a la poda y aspectos técnicos necesarios. Priorizar la seguridad del parque con la finalidad de garantizar la protección y su cuidado y fomentar hábitos de conciencia ambientalista en cada una de las personas que habitan en la comunidad del parque, logrando recuperar y mantener las áreas verdes. Estas fueron las estrategias exitosas utilizadas para la recuperación de las áreas verdes del parque.

\section{MATERIALES Y MÉTODOS}

Para el desarrollo de la investigación se utilizaron métodos de investigación científica como:

Del nivel teórico:

Histórico - lógico: se usó en la construcción de la investigación para la indagación de los antecedentes.

Análisis - síntesis: Se utilizó para diagnosticar los problemas que atentaban contra el parque y sus áreas verdes.

Del nivel Empírico:

Observación: permitió investigar y analizar la mayor cantidad de información, dando las pautas necesarias para el desarrollo de la investigación.

Entrevista: Brindo soluciones significativas al desconocimiento que tenían varios vecinos de la importancia del cuidado y protección del parque de la ciudadela 6 de Agosto.

Revisión bibliográfica: Se utilizó para la recopilación de información relacionada al tema, mediante libros, revistas de carácter científico, internet, entre otros.

14 UNESUM-Ciencias. Publicación cuatrimestral. Vol. 3, Año 2019, No. 3 (Septiembre - Diciembre) 


\section{Resultado}

Se llegó al siguiente resultado con el conocimiento adecuado sobre la importancia del cuidado a los parques y a las áreas verdes se fomentaron hábitos de conciencia ambientalista en las personas que habitan en la comunidad del parque, logrando recuperar las áreas verdes y desarrollando el trabajo de forma creativo, para un buen vivir sano. En la actualidad se puede observar como el parque es visitado y el disfrute de sus visitantes. El embellecimiento del parque 6 de agosto fue una experiencia que debe propagarse por varios parques de la ciudad.

\section{CONCLUSIONES}

Se concluye que en la actualidad la presencia de áreas verdes en un espacio urbano es considerada importante, pues su acción sobre el ambiente es positiva. Se conoce que la presencia de una concentración forestal, dentro de la ciudad significa, una actividad de oxigenación del ambiente, lo que contribuye a reducir la contaminación ambiental, por otra parte aumenta la humedad del aire. La población urbana es amante de los espacios abierto como parques y jardines, estos les proveen salud, bienestar y muy importante la incorporación a la sociedad a través de deportes o recreación sana. Las capacitaciones favorecieron en cuanto al cuidado y preservación del parque de la ciudadela 6 de Agosto del cantón Jipijapa, hoy se cuenta con un grupo de personas de todas las edades que están al pendiente de su cuidado y lo más relevante es que le educan a los niños pequeños en cuanto a la importancia del cuidado de las áreas verdes y al medio ambiente.

\section{REFERÉNCIAS BIBLIOGRÁFICAS}

Anaya, José María Chávez. «Verde Bandera. Periodismo Ambiental / Guadalajara - México.» Editado por Sergio Hernández - MURAL. 13 de Abril de 2008.

Borja, Jordi. El espacio público: ciudad y ciudadanía. Barcelona.: Editorial: MUXI, Zaida, 2001.

Corraliza, Rodríguez José Antonio. «Emoción y espacios públicos: La experiencia humana de los escenarios urbanos.» Jornada de El árbol en el diseño urbano. Madrid, Santa \& Cole: Universidad Autónoma de Madrid., 2009.

Falcón, Antoni. Espacios Verdes para una ciudad sostenible: Planificación, proyecto, mantenimiento y gestión. Barcelona: Editorial: Gustavo Gili, SL., 2007.

Lynch, Kevin. Planificación del sitio. Traducido por Arq. Julia Fernandez de Caleya. Barcelona: Editorial: Gustavo Gili, S. A., 1980.

OMS, Organización Mundial de la Salud. Promoción de la Salud. Glosario, Ginebra: World Health Organization (WHO), 1998.

Palomo, Pedro Jose Salvador. La Planificación verde en las ciudades. Barcelona: Editorial: Gustavo Gili, SA., 2003.

Presidencia de la Republica. «Sistema Internet de la Presidencia 2007.» 2007.http://pnd.calderon.presidencia.gob.mx/pdf/Eje4_Sustentabilidad_Ambiental/eje_4_ Sustentabilidad_Ambiental.pdf (último acceso: 22 de Mayo de 2009). 
Marleydis Claudia Solís Maldonado, Kirenia Maldonado Zuñiga, Dunia Lisbet Domínguez Gálvez...

Ramos, Jesús Aantiago. «El papel del sistema de espacios verdes en la multifuncionalidad del PaisajeUrbano. Aplicacion al Area Metropolitana de Sevilla.» Editado por Asociación de Portuguesa de Geógrafos. X Colóquio Ibérico de Geografia. Universidade de Évora, 2005.

Shilling, J. D. (1992). Hedonic prices and contractual contingencies. Journal of Urban Economics, 32(1), 108-118. doi: 10.1016/0094-1190(92)90017-F [ Links ]

Smith, V. K., \& Huang, J. C. (1995). Can markets value air quality? A meta-analysis of hedonic property value models. Journal of Political Economy, 103(1), 209-227. Retrieved from http://thacher.us/jenn/econ542/articles/smith1995.pdf [ Links ]

Stronegger, W. J., Titze, S., \& Oja, P. (2010). Perceived characteristics of the neighborhood and its association with physical activity behavior and self-rated health. Health \& Place, 16(4), 736-743. doi: 10.1016/j.healthplace.2010.03.005 [ Links ]

Takano, T., Nakamura, K., \& Watanabe, M. (2002). Urban residential environments and senior citizens' longevity in megacity areas: The importance of walkable green spaces. Journal of Epidemiology and Community Health, 56, 913-918. doi: 10.1136/jech.56.12.913 [ Links ]

Torres, Y., \& Arranz, J. J. (2015). Técnicas de teledetección y análisis especial aplicadas a la agricultura urbana. En J. Briz, \& I. de Felipe (Eds.), Agricultura urbana integral: ornamental y alimentaria (pp. 417-426). Madrid, España: Ministerio de Agricultura, Alimentación y Medio Ambiente. [ Links ]

Tyrvainen, L., \& Miettinen, A. (2000). Property prices and urban forest amenities. Journal of Environmental Economics and Management, 39(82), 205-223. doi: 10.1006/jeem.1999.1097 [ Links ]

Yamamoto, K. (2010). Evaluation of the degree of sufficiency of public green spaces as an indicator of urban density in metropolitan areas in Japan: The Chubu metropolitan area. International Journal of Environmental, Chemical, Ecological, Geological and Geophysical Engineering, 4(7), 276-284. Retrieved from https://scholar.waset.org/1999.6/4711 [ Links ] 